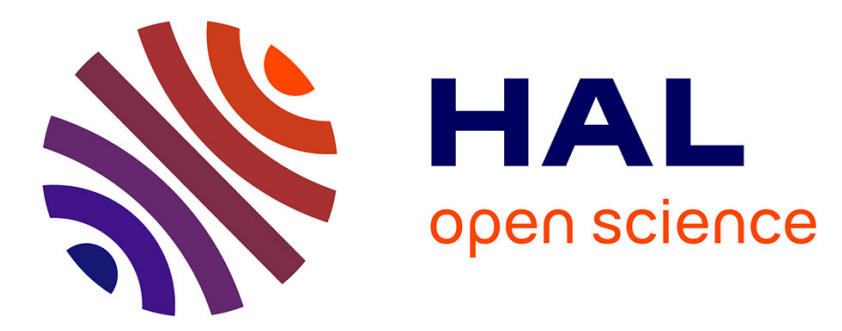

\title{
Contact of a Finger on Rigid Surfaces and Textiles: Friction Coefficient and Induced Vibrations
}

Ramona Fagiani, Francesco Massi, Eric Chatelet, Jean-Philippe Costes, Yves Berthier

\section{- To cite this version:}

Ramona Fagiani, Francesco Massi, Eric Chatelet, Jean-Philippe Costes, Yves Berthier. Contact of a Finger on Rigid Surfaces and Textiles: Friction Coefficient and Induced Vibrations. Tribology Letters, 2012, 46 (3), pp.1-16. 10.1007/s11249-012-0010-0 . hal-01087044

\section{HAL Id: hal-01087044 https://hal.science/hal-01087044}

Submitted on 25 Nov 2014

HAL is a multi-disciplinary open access archive for the deposit and dissemination of scientific research documents, whether they are published or not. The documents may come from teaching and research institutions in France or abroad, or from public or private research centers.
L'archive ouverte pluridisciplinaire HAL, est destinée au dépôt et à la diffusion de documents scientifiques de niveau recherche, publiés ou non, émanant des établissements d'enseignement et de recherche français ou étrangers, des laboratoires publics ou privés. 


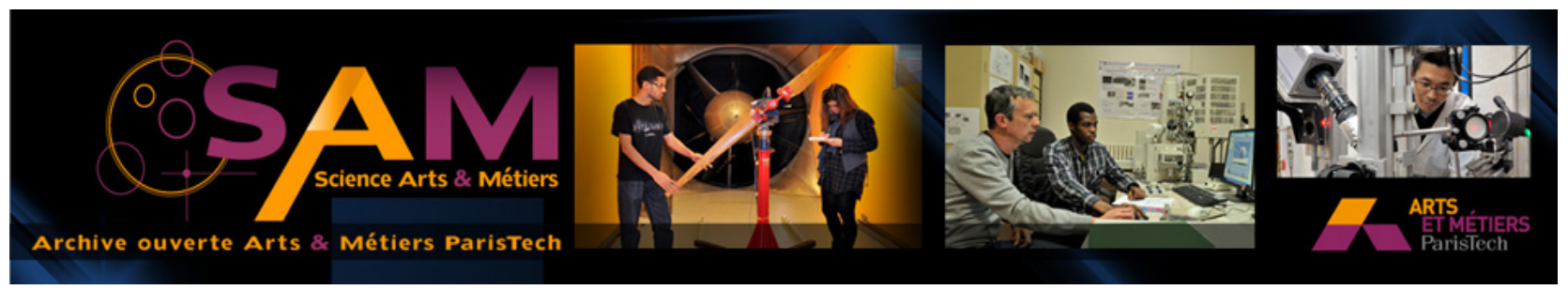

Science Arts \& Métiers (SAM)

is an open access repository that collects the work of Arts et Métiers ParisTech researchers and makes it freely available over the web where possible.

This is an author-deposited version published in: http://sam.ensam.eu

Handle ID: .http://hdl.handle.net/10985/8982

\section{To cite this version :}

Ramona FAGIANI, Francesco MASSI, Eric CHATELET, Jean-Philippe COSTES, Yves BERTHIER - Contact of a Finger on Rigid Surfaces and Textiles: Friction Coefficient and Induced Vibrations - Tribology Letters - Vol. 46, n³, p.1-16 - 2012 


\title{
Contact of a Finger on Rigid Surfaces and Textiles: Friction Coefficient and Induced Vibrations
}

\author{
Ramona Fagiani - Francesco Massi • \\ Eric Chatelet $\cdot$ Jean Philippe Costes • \\ Yves Berthier
}

\begin{abstract}
The tactile information about object surfaces is obtained through perceived contact stresses and frictioninduced vibrations generated by the relative motion between the fingertip and the touched object. The friction forces affect the skin stress-state distribution during surface scanning, while the sliding contact generates vibrations that propagate in the finger skin activating the receptors (mechanoreceptors) and allowing the brain to identify objects and perceive information about their properties. In this article, the friction coefficient between a real human finger and both rigid surfaces and fabrics is retrieved as a function of the contact parameters (load and scanning speed). Then, the analysis of the vibration spectra is carried out to investigate the features of the induced vibrations, measured on the fingernail, as a function of surface textures and contact parameters. While the friction coefficient measurements on rigid surfaces agree with empirical laws found in literature, the behaviour of the friction coefficient when touching a fabric is more complex, and is mainly the function of the textile constructional properties. Results show that frequency spectrum distribution, when touching a rigid surface, is mainly determined by the relative geometry of the two contact surfaces and by the contact
\end{abstract}

R. Fagiani · F. Massi $(\bowtie) \cdot$ E. Chatelet · Y. Berthier Université de Lyon, CNRS, INSA-Lyon,

LaMCoS UMR5259, 69621 Lyon, France

e-mail: francesco.massi@insa-lyon.fr

R. Fagiani

Department of Mechanical and Aerospace Engineering, University of Rome 'La Sapienza', via Eudossiana 18, 00184 Rome, Italy

J. P. Costes

Ecole Nationale Supérieure d'Arts et Métiers, LaBoMaP, rue Porte de Paris, 71250 Cluny, France parameters. On the contrary, when scanning a fabric, the structure and the deformation of the textile itself largely affect the spectrum of the induced vibration. Finally, some major features of the measured vibrations (frequency distribution and amplitude) are found to be representative of tactile perception compared to psychophysical and neurophysiologic works in literature.

Keywords Tactile perception - Mechanoreceptors ·

Textiles $\cdot$ Friction-induced vibrations $\cdot$ Friction coefficient

\section{Introduction}

During the contact, the human hand is able to detect different object properties and characteristics such as material, geometry and temperature. Different recognised properties are linked to different hand motions. The specific movements made by either the hand (active touch) or the object (passive touch) activate the somatosensory neurons that innervate the skin, providing the connection between touch and object properties perception. The somatosensory neurons transduce the physical stimulus into an electric one sent to the brain.

The cutaneous sensory neurons can be divided into three classes according to sensory modality $[1,2]$ : nociceptive units, thermoreceptive units and mechanoreceptive units. While the two last afferents take their name from the stimulus they are most sensitive to, the nociceptive units are able to respond to both the mechanical and thermal stimuli but from a very high threshold, corresponding to a damaging level and thus to pain sensations [3]. Thermal information, in the range of $5-45^{\circ} \mathrm{C}$, is transduced from the thermoreceptors that are divided into warm and cold receptors [4]. 
Focusing the attention on the touch sensation, it is necessary to investigate the role of the mechanoreceptive units that are activated by the skin stress-state variation generated during the tactile sensing. In fact, due to the mechanical loads generated by the contact, the fingertip skin surface is deformed. The space-time variation of the skin stress-state causes the mechanoreceptors to respond with an appropriate space-time variation of their discharge rate. In this way, the mechanical stimulus (deformations and vibrations induced from the finger-surface scanning and propagating in the skin) is transduced into neural signals.

These tactile units are made by an afferent fibre, and its unmyelinated ending, that gives the possibility to distinguish them as Merkel disks, Meissner's corpuscles, Ruffini endings and Pacinian corpuscles (Fig. 1). They are located at different depths in the skin, ranging from the epidermis (Meissner's corpuscles) to the subcutaneous fat layer, corresponding to a maximal depth of about $3 \mathrm{~mm}$ (Fig. 1).

As indicated in [5, 6], four different tactile afferent populations are associated with these endings. On the basis of their receptive field size and their response to sustained stimulation, it is possible to classify the tactile afferent populations in: SA I (slowly adapting type I), FA I (fast adapting type I), FA II (fast adapting type II) and SA II (slowly adapting type II).

Their innervation density is not uniform, since it changes in the function of site and receptor type, conferring different sensitivity from a zone to another and between different stimuli, depending also on gender, age, health status, etc.

The classification criteria are based on the hypothesis that the ending structure is linked to its sensory function and that its location corresponds to the maximum

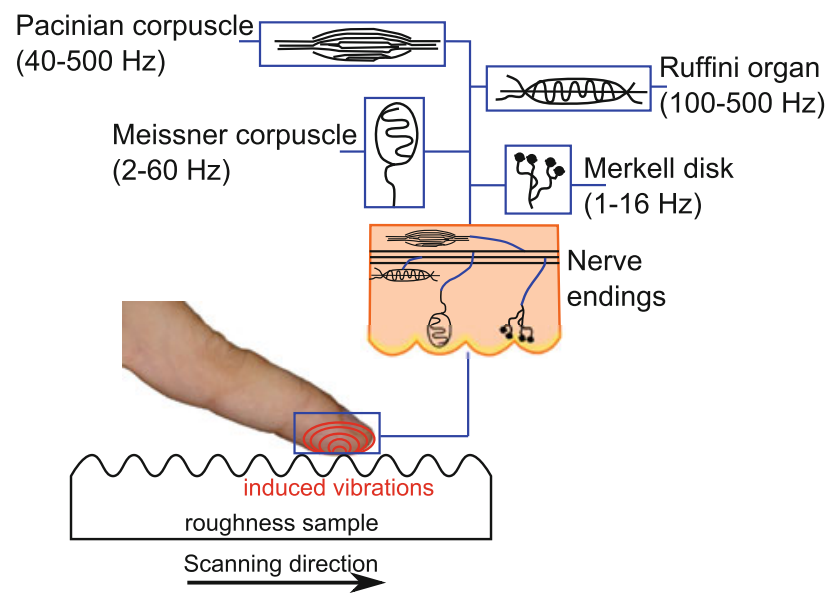

Fig. 1 Schematic representation of the contact between finger and object surface, and scheme of the mechanoreceptors position into the skin. The scanning direction is along the line of the finger (from left to right, with respect to the figure) sensitivity area. The first assumption has been demonstrated by the study of the receptive fields [1], while the second is confirmed by numerical models highlighting that the higher strain energy is concentrated at the tactile receptor locations [7].

SA I reacts from about $2-16 \mathrm{~Hz}$, and it is responsible for the detection of the spatial structure and the shape of an object [8-12]; FA I, easily excited between $3 \mathrm{~Hz}$ and $40 \mathrm{~Hz}$, gives the perception of the motion (fundamental for grip control) [8, 13]; FA II, reacting in a range of 40-500 Hz, transmits vibration sensation, and SA II [6, 14], responding from 100 to $500 \mathrm{~Hz}$, gives the skin stretch sensation [15-19].

The relative motion between a surface and a finger under adequate applied force and scanning speed enables to perceive the surface characteristics.

In recent psychophysical and neurophysiologic works, the perception mechanism is ascribed to the "duplex model of tactile roughness perception" [19-31]. From this point of view, the perception of fine textures (spatial period is smaller than $100 \mu \mathrm{m}$ ), perceived by the induced vibrations, is different from the perception of coarse textures (spatial period is bigger than $200 \mu \mathrm{m}$ ), characterised by a "single spatial intensive code" and mediated by SA I afferents. This theory has been supported experimentally in a previous work [32].

In this context, it is necessary to perform appropriate experiments to find out frictional properties and features of the vibrations induced by surface scanning. While the friction aspects of the scanning finger-surface contact are quite well investigated (several studies on the effects of loads $[33,34]$, pressure, finger inclination with respect to the surface, and moisturisers $[35,36]$ are reported in the literature), the induced vibration spectra and the consequent activation of the mechanoreceptors on the skin were rarely investigated.

The analysis of friction properties and vibration spectra, induced by surface scanning, can contribute to the definition of objective indexes about surface perception (softness, textile quality, perceived roughness, etc.). In this article, experimental tests characterising friction coefficient and vibration spectra with respect to scanning speed, contact load and surface roughness on both rigid surfaces and textiles are presented and linked to the surface and textile properties.

\section{Experimental Test Bench}

\subsection{TriboTouch Set-Up}

The behaviour of the right-hand index finger scanning on rigid aluminium surface samples and on fabric samples is investigated. Using an innovative test bench, named 


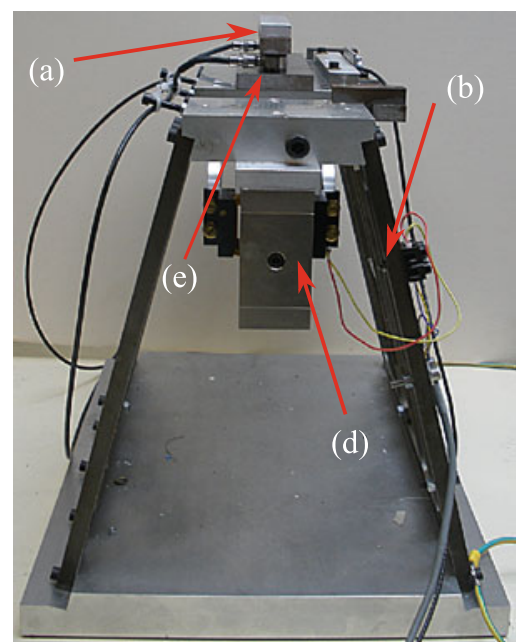

(A)

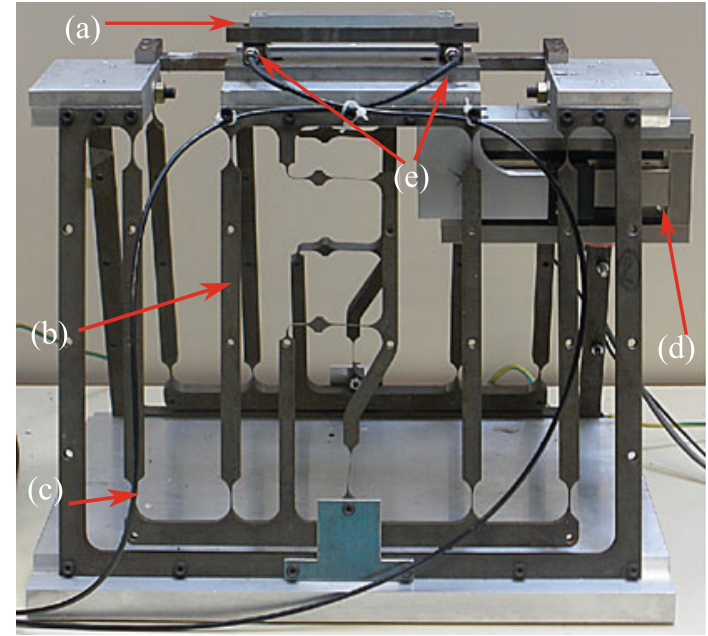

(B)

(a) Surface sample;

(d) Linear voice coil actuator;

(b) Compliant mechanisms; (e) Force transducers;

(c) Flexible joint;

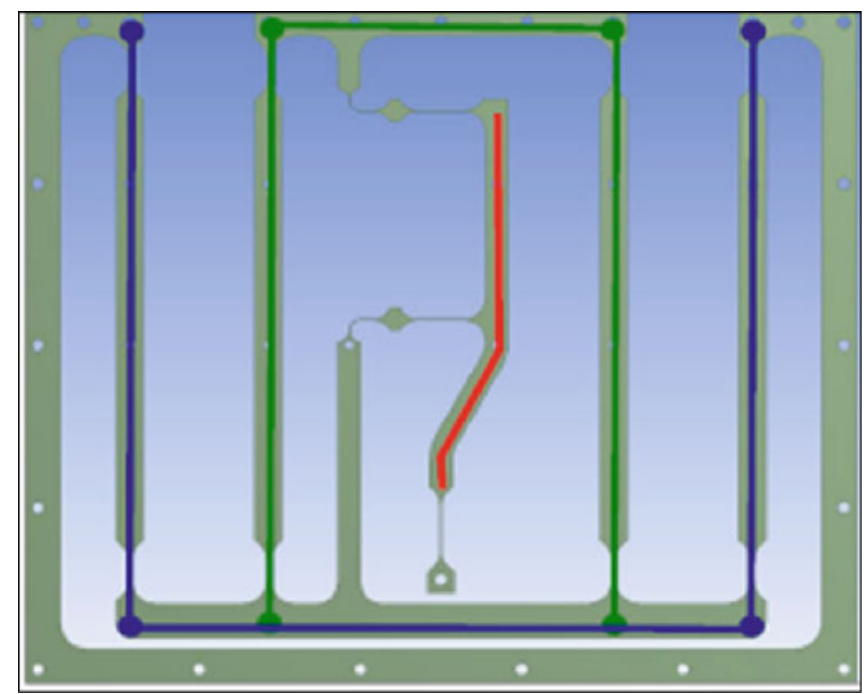

(C)

Fig. 2 Front (a) and side (b) view of the experimental set-up (scale 1:8), and a scheme of the compliant system (c)

TriboTouch (Fig. 2), it has been possible to reproduce the finger-surface scanning and analyse the influence of scanning speed, surface texture and contact force on the vibration measured on the fingertip and on the friction coefficient.

The reproduction and the analysis of finger-surface scanning involves difficulties that are related to the material characteristics and the measurements themselves. In fact, it is impossible to measure the vibrations directly at the contact region between the skin and the sample surface without modifying the contact itself, and it is extremely difficult to isolate such low amplitude vibrations from the parasitic noise coming from the set-up.
For this reason, the TriboTouch set-up (Fig. 2) has been developed to reproduce the finger-surface scanning phenomena under the real values of the contact features (scanning speed, contact forces, surface texture, etc.), guaranteeing measurements reproducibility and avoiding noise generation. The sample surface (a) is translated by a compliant mechanism (b), which allows linear motion of the sample without the involvement of any other sliding contact. The compliant guide is a mechanism consisting of rigid links connected to one another by flexure hinges (c).

For system stability, two nominally identical compliant mechanisms are placed symmetrically respect to the median plane and slightly tilted toward each other, as shown in 
Fig. 2. Each element is a double parallelogram that allows for a linear translation of the upper rigid segment where the sample is mounted (a). Indeed, while the lower horizontal segment of the first parallelogram (blue) performs a circular trajectory, the vertical displacement is retrieved by the trajectory of the upper basis of the second parallelogram (green). The same deformation for the two parallelograms, necessary for achieving a horizontal trajectory of the upper segment, is guaranteed by the central lever (red in Fig. 2) that connects the two horizontal segments and rotates with respect to the lowest point that is fixed to the frame. The flexible joints (c) approximate pin joints between the segments of the double parallelograms and are designed to allow for a linear displacement of the surface sample of up to $100 \mathrm{~mm}$. The horizontal translation of the sample is realised by a linear voice coil actuator (d), which allows to impose the desired scanning velocity through a feed-back control and a TTL linear encoder.

The linear voice coil actuator is a linear motion device based on a magnetic field and a coil winding to generate a force which is proportional to the current applied. The coil is free to move axially in the air gap between a centre pole and a permanent magnet surrounding it. An iron shell houses both the magnet and the pole. The relative motion between the coil and the magnet is thus obtained without any contact.

In this manner, the sample is moved without generating noise (undesired vibrations) because the only surfaces in sliding contact is the one between the finger and the sample, while the compliant system transfers the motion gaining its mobility from the deflection of the flexible members rather than from the sliding joints. The flexure hinge, a thin member that provides relative motion between two adjacent rigid members through flexing [37], is obtained by machining a blank piece of material, with no need for any sliding surface and consequently without any friction losses or noise from the joints.

Two triaxial force transducers (e), detecting normal and tangential forces, are placed below the sample for measuring the contact forces. To have a better control of the contact force, during sample scanning, the fingertip is fixed, while the scanned surface is moved by the sample translation. The individual stays close to the experimental set-up, leaning his elbow on a rigid planar surface to limit unintentional movements.

A small accelerometer is mounted on the finger nail to measure the vibrations induced by the relative motion between finger and test surface (Fig. 3). The negligibility of the measurement error introduced by the added mass of the accelerometer was previously verified by comparing the signal obtained by the accelerometer to the one detected with a laser vibrometre [38].

Preliminary tests have been carried out to verify that the proposed set-up was able to detect the desired vibrations

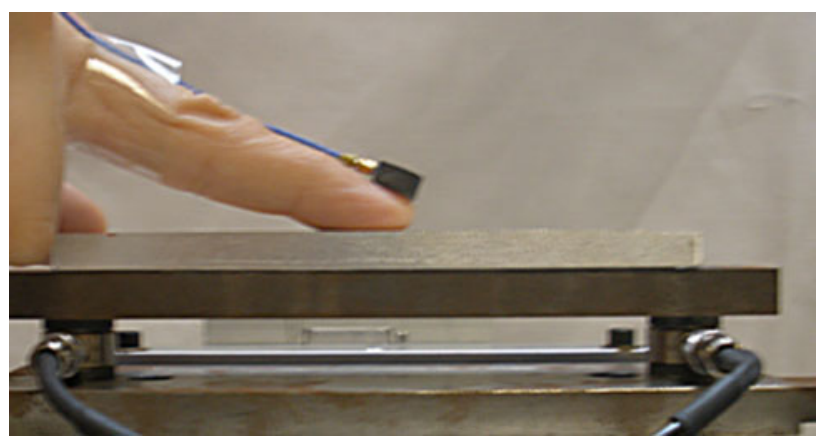

Fig. 3 Experimental set-up configuration used for measurements

and to verify the negligible value of the parasitic noise coming from the set-up operation [32].

\subsection{Materials and Methods}

The scanning of a human finger on rigid surfaces and textiles is reproduced. The normal and tangential forces measured by the triaxial force transducers are used to calculate the macroscopic friction coefficient during surface scanning. The surface sample accelerates to reach the desired velocity that is maintained constant throughout the test. The value of the friction coefficient is calculated at constant sliding speed, while the normal load is monitored. Only tests at constant normal load are retained for the analysis. A frequency analysis of signals measured by the accelerometer during the surface scanning is carried out to investigate the vibration spectra and to highlight their features as a function of contact parameters such as scanning speed, contact load, surface roughness and surface texture. To identify the main features of the frequency distribution of the measured spectra, measurements on surface samples with periodical roughness (Fig. 4) and on textiles are performed.

The nine surface samples with periodical roughness involved in the measurements are obtained by milling the surfaces with different milling cutters [32]. Large values of the cutter radii have been chosen to minimise the curvature of the roughness ridges.

The chosen roughness values (ranging from 0.64 to $5.2 \mu \mathrm{m})$ represent common surfaces perceived as practically smooth and with a well-defined roughness. For example, with respect to commonly used objects,

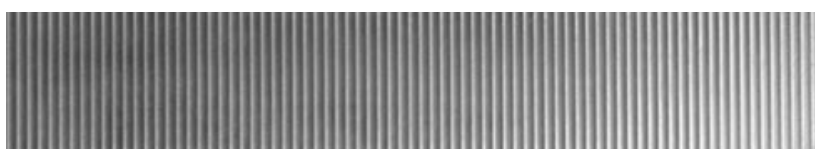

Fig. 4 Example of aluminium surface sample obtained by milling. This sample has a roughness period of $1.4 \mathrm{~mm}$ and a mean roughness of $3.7 \mu \mathrm{m}$ 
$R_{\mathrm{a}}=0.64 \mu \mathrm{m}$ is the roughness of an aluminium can, and $R_{\mathrm{a}}=5.2 \mu \mathrm{m}$ is a type of bottle cork.

In order to have a wide range of textures, the chosen fabric samples differ from one another in terms of material, texture and hairiness.

The roughness parameters of the rigid surface samples have been verified by a laser profilometer, while the fabric features have been observed by an optical microscope.

The considered scanning speeds are 10,20,30, 40 and $50 \mathrm{~mm} / \mathrm{s}$. Each surface sample is investigated at each scanning speed for different individuals.

All the experiments take place at the same temperature of $24{ }^{\circ} \mathrm{C}$ and in the same environmental conditions.

The skin is untreated, meaning that no moisturisers are employed. The finger and the surface are cleaned with alcohol before starting with measurements. The accelerometer detecting the induced vibrations is attached to the finger nail by means of wax. The finger is put in contact with the surface sample and then the sample starts translating. The scanning direction is the direction on the line of finger bones, perpendicular to the fingerprint direction.

\section{Friction Coefficient}

Stretching of the fingertip skin, occurring when sliding on a surface, is largely affected by the friction coefficient, which consequently affects the mechanoreceptor activation and the amplitude of the induced vibrations (a larger magnitude of the friction forces corresponds to a larger amount of energy transferred to the generated acoustic field). For these reasons, particular attention has been focused on the measurement of the friction coefficient between the fingertip and the tested surfaces. The TriboTouch system is able to detect the normal $(N)$ and tangential $(T)$ loads by means of the triaxial force transducers during contact, allowing to determine the macroscopic friction coefficient $(\mu)$ :

$\mu=\frac{T}{N}$

Tests have been performed to characterise the friction coefficient and its changes as a function of scanning speed and normal load, when scanning both aluminium samples and textiles. The skin is investigated in untreated conditions, meaning that the finger is cleaned with alcohol and dried before each test, so that the measured friction coefficient reflects comparable skin hydration state and lipid concentration.

Figure 5 shows an example of the friction coefficient obtained by scanning the finger on an aluminium surface sample (periodic roughness period $0.3 \mathrm{~mm}$ and mean roughness $4 \mu \mathrm{m}$ ) as a function of the applied load and for

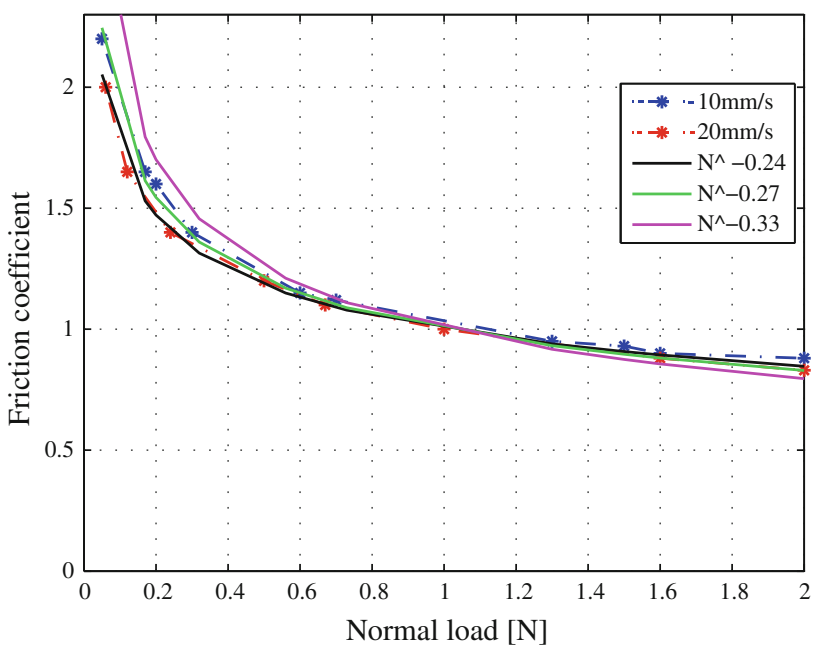

Fig. 5 Normal load effect on the friction macroscopic coefficient between fingertip and aluminium samples for different scanning speeds. Experimental results (dashed lines) for 10 and $20 \mathrm{~mm} / \mathrm{s}$, and analytical curves for different exponents of the normal load

different scanning speeds. The friction coefficient decreases as the contact force increases, highlighting that the skin/surface contact does not adhere to the Amonton's law $[39,40]$. In fact, the skin relief deformations are not negligible and, as the load increases, these deformations rise until the reliefs are completely deformed and the contact area approximates the contact of a large single relief [35, 36, 41]. The difference with the Amonton's law has been explained in literature $[35,36,41]$ by the difference between the theoretic and the actual contact area (because of skin ridges or, more in general, of skin topography), and its nonlinear growth with the load applied. Moreover, when the sliding contact takes place, the skin is deformed and a series of "lines" called reliefs appear, as a function of load and sliding speed. These reliefs are closed together by compression and then opened back once they enter the contact zone.

Several authors [33-36, 41, 42] have developed skin friction analysis supporting the Wolfram theory for a sliding surface on a distorting one (developed on the basis of the Hertz contact model to account for adhesion that mainly affects the skin friction), which allows to calculate the friction coefficient as:

$\mu \alpha S\left(\frac{K}{E}\right)^{2 / 3} N^{-1 / 3}$

where $N$ is the applied load, $E$ is the Young's modulus of the skin, $K$ is a colligative term including average dimension of adhesive contacts, their number and frequency per unit areas, and $S$ is the shear strength of the adhesive contacts. 
These experimental works, consistent with the adhesion model, show little variation in the load power value related to the experimental conditions and the body site where the skin is analyzed. Specifically, the Wolfram theory suggests that $\mu$ is proportional to $N^{-0.33}$, while e.g. Sivamani et al. [36] found out a dependence on $N^{-0.32}$ for dorsal skin of middle finger, and Koudine et al. [41] $N^{-0.28}$ for forearm skin.

The results presented in Fig. 5 show a proportionality of the friction coefficient to a power of the normal load varying from -0.27 (for a scanning speed of $10 \mathrm{~mm} / \mathrm{s}$ ) to -0.24 (for a scanning speed of $20 \mathrm{~mm} / \mathrm{s}$ ), for the glabrous skin of the fingertip.

Experiments show that the macroscopic friction coefficient between skin and aluminium surface is slightly affected by speed variations (Fig. 6), which may be mainly ascribed to the decrease of the viscosity of the interface layer on the skin with the increase of the shear rate due to natural skin lipid film and hydration [33].

While the surface of an aluminium sample can be considered as rigid with respect to the fingertip, when scanning on a textile both surfaces are deformed. A different behaviour of the friction coefficient with respect to sliding speed and normal load is expected.

Figures 7 and 8 show the friction coefficient as a function of the scanning speed when the finger scans on fabrics with a well-defined texture periodicity and small yarn roughness (Fig. 7) and on fabrics with high hairiness (Fig. 8).

With reference to the effect of the scanning speed on the friction coefficient, it is possible to assert that in every case, the friction coefficient tends to decrease as the scanning

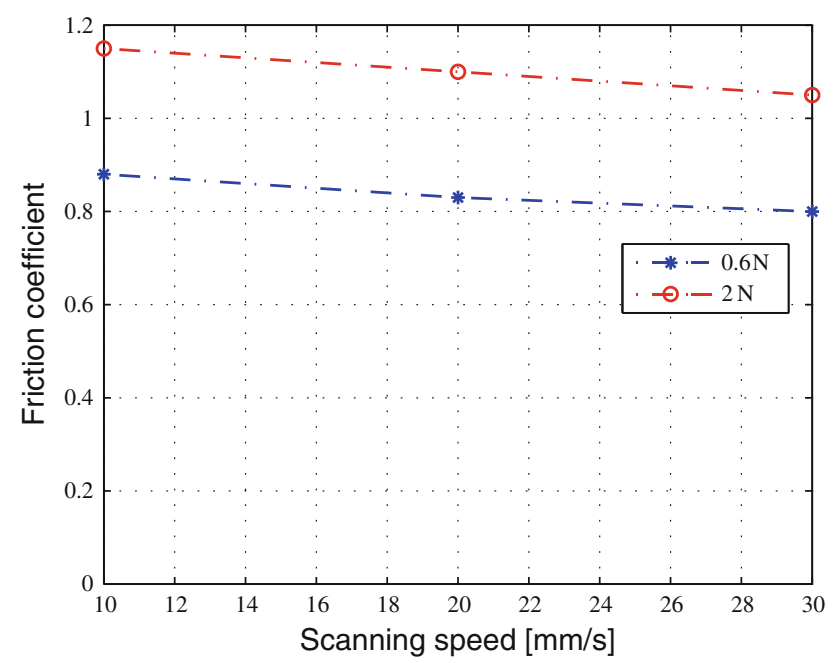

Fig. 6 Scanning speed effect on the friction macroscopic coefficient between fingertip and aluminium samples for two different contact loads speed increases. When dealing with textile, the friction coefficient decrease seems to be largely affected by the fabrics yarn characteristics: fabrics with well-defined texture periodicity and small yarn roughness show a smaller decrease of the friction coefficient with respect to the scanning speed (Fig. 7), similarly to the scanning on aluminium samples (Fig. 6). On the contrary, fabrics with high hairiness show large variations of the friction coefficient (Fig. 8). The higher is the hairiness, the larger is the variation of the friction coefficient with the scanning speed (as shown by the increasing hairiness of the tissues (a), (b) and (c) in Fig. 8). This different behaviour can be ascribed to the fact that, when scanning on a rigid sample, the slight variation of the friction coefficient is mainly due to the finger compliance and to its moisture, while when dealing with textiles, it is necessary to account for the textile compliance and accommodation phenomena. These results are in contrast with the experiments presented in [43] which show a friction coefficient which increases with the scanning speed. Nevertheless the difference could be ascribed to the different velocity range and to the use of a polymeric fake skin in [43], instead of a real human finger. The results presented here show that in the fabric-skin contact, the yarn hairiness plays a major role in the friction process allowing for larger values of the friction coefficient at low sliding speeds (tissues (b)nd (c) in Fig. 8).

Looking at the effect of the contact load on the friction coefficient, as shown by the comparison between Figs. 5 and 9, the trend of the friction coefficient between fingertip and fabrics is more complex. For both fabrics with a regular texture (blue line in Fig. 9) and fabrics with high hairiness (red line), the friction coefficient has large variations for low contact forces (Fig. 9). This behaviour could be ascribed to the yarn's and fibre's deformations and to the interlocking of the fibre hairs with the fingerprint ridges. Fabrics with high hairiness show larger values and larger variations of the friction coefficient at low contact load, because of the need to deform the fabric hairiness when sliding with the fingertip. This trend changes and the friction coefficient becomes almost constant when increasing the normal load over a critical value (about $1 \mathrm{~N}$ ) for both kinds of fabric. This behaviour is due to the deformation of fabrics that reaches a limit value together with the effective contact area and the surface roughness.

\section{Induced Vibrations}

In previous works, experimental campaigns have been carried out to validate the TriboTouch set-up [32], the measurements methodology [38], and to investigate the influence of scanning speed, surface roughness, and texture on the vibration spectra. 
Fig. 7 Scanning speed effects on the friction coefficient between finger and fabric samples with regular texture. The fabric figures are in scale 13:1

Fig. 8 Scanning speed effects on the friction coefficient between finger and fabric samples without a regular texture and with a high hairiness. The fabric figures are in scale 13:1

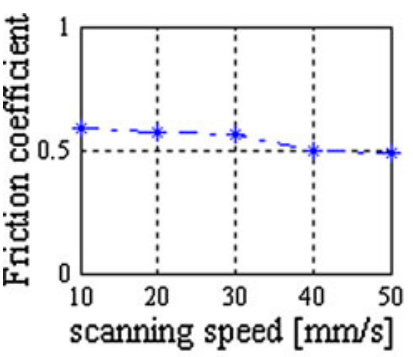

(a)
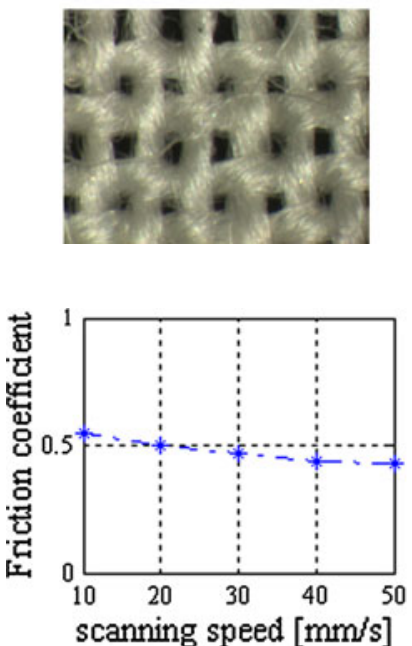

(a)

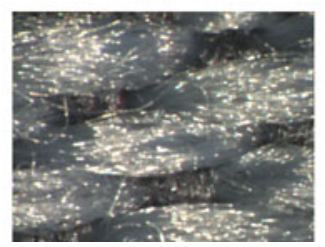

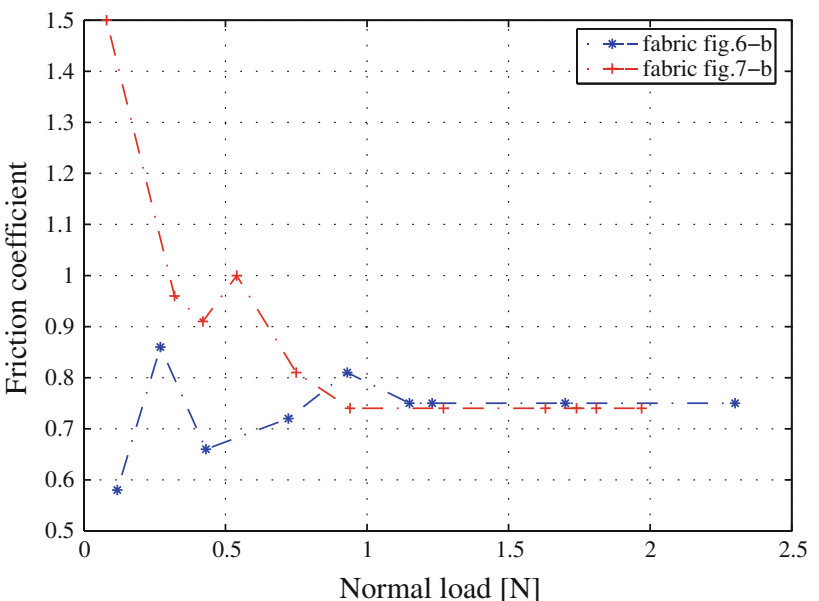

Fig. 9 Contact load effects on the friction coefficient between finger and fabric samples for a scanning speed of $10 \mathrm{~mm} / \mathrm{s}$

The tests have been developed with different individuals (different fingerprints) on periodic surface samples with different roughness spatial periods and on surfaces with isotropic

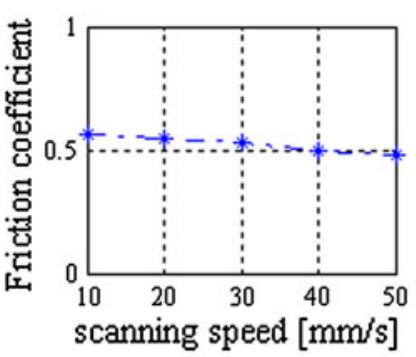

(b)
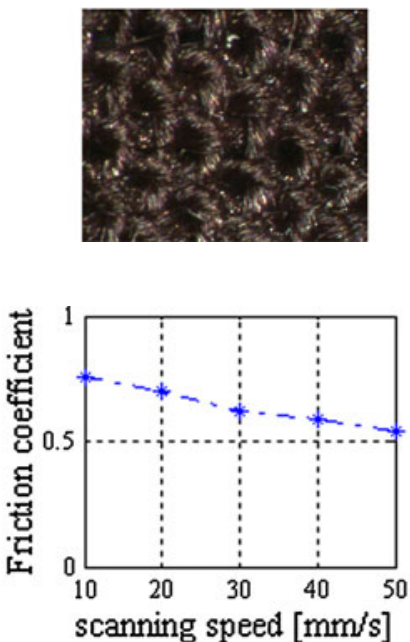

(b)

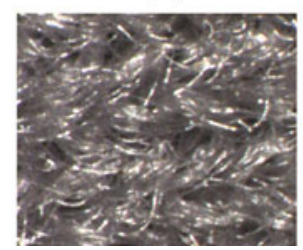

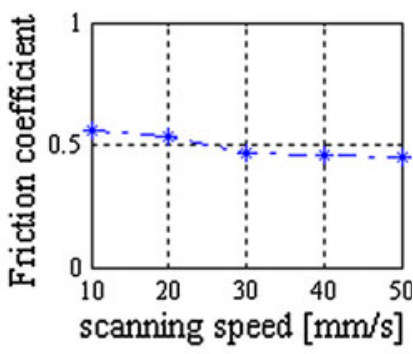

(c)
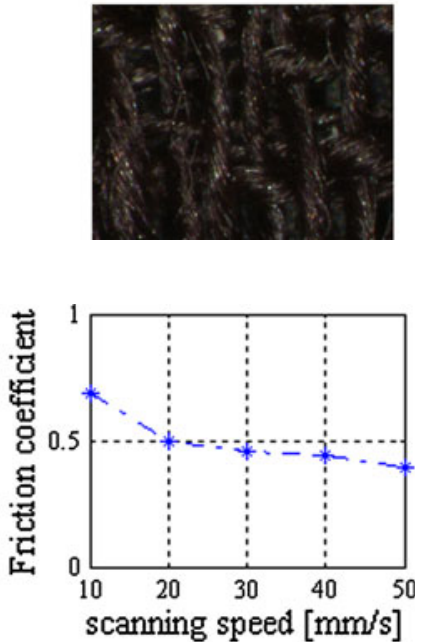

(c)

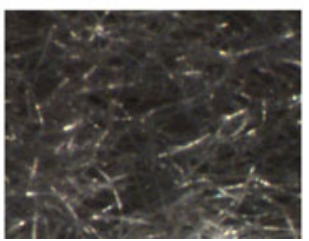

roughness [32]. In the following section, a simple numerical model is presented and used to attribute the frequency distribution retrieved on periodical surfaces [32] to the mutual geometry of the surfaces in contact. Then, the effect of the normal load on the vibration amplitude is investigated. Finally the vibration spectra arising from the scanning of fabric samples with different texture and hairiness is presented.

\subsection{Fingerprints-Periodic Samples Contact Model}

Measurements have been performed on aluminium surface samples with periodical roughness (roughness spatial period varying between 0.15 and $2.17 \mathrm{~mm}$ ) [32, 38]. In these cases, the vibration spectrum is characterised by a welldefined frequency peak [32], which is a function of the scanning speed and of the relationship between the roughness spatial period (width) of the surface samples and of the fingerprint. With respect to the scanning speed, the frequency of the induced vibrations increases linearly as the scanning speed increases [32]. 
With respect to the sample roughness width (spatial period), the frequency of the induced vibrations is affected by the sample surface width only when the texture width is smaller or comparable to that of the fingerprints. When the sample surface period is larger than the fingerprints, the frequency of the induced vibrations is only function of the fingerprints width.

These results agree with the duplex model of tactile perception, for coarse and fine textures, highlighted by psychophysical and neurophysiologic studies available in literature [20-23].

The same behaviour is found in different individuals; nevertheless, even if the global trend of the spectra in function of roughness and scanning speed is the same, the values of the frequency peak change because of the different finger and fingerprint characteristics.

To better investigate this phenomenon, it is possible to consider a simple numerical model able to reproduce the interaction between two sinusoidal surfaces in relative motion. In this way, it is possible to investigate the geometrical effect of the roughness on the induced vibration frequencies only, neglecting all the material and contact parameters. The aim is to highlight whether the trend of the vibration frequency is a function of the mutual geometry of sample and fingerprints roughness.

The model has been developed with Matlab to be representative of the vibrations induced by the interaction between a compliant sinusoidal surface (fingerprints) and a rigid one (aluminium sample) as a function of the roughness wavelength for different scanning speeds. The model does not account for the material and contact properties.

The main hypothesis is that the skin at the fingerprint deforms to follow the profile of the aluminium surface, i.e. the local deformation of the skin is proportional to the intersection area between the two sinusoids (Fig. 10). Thus, the compliance of the fingertip skin (with respect to the aluminium) and its local deformation (which is directly linked to the local vibration) is approximated by the intersection area (red area in Fig. 10).

Both the fingerprint profile $(w l)$ and the surface sample profile (w2) are modelled as sinusoids (Eq. 3). The sinusoidal roughness profiles are created using the arithmetic mean values of the wavelength $\left(l_{2}\right)$ and roughness $\left(R_{2}\right)$ of the periodical surface samples used for the experimental tests. The Matlab model simulates the horizontal ( $x$-direction) relative motion at a constant speed $(v)$ of the sinusoid representing the fingerprint $(w l)$ with respect to the surface sample sinusoid $(w 2)$.

$$
\begin{aligned}
w 1(t, x) & =R_{1} \sin \left(\frac{2 \pi}{\lambda_{1}} \cdot x+v \cdot t\right), \\
w 2(x) & =R_{2} \sin \left(\frac{2 \pi}{\lambda_{2}} \cdot x\right)
\end{aligned}
$$

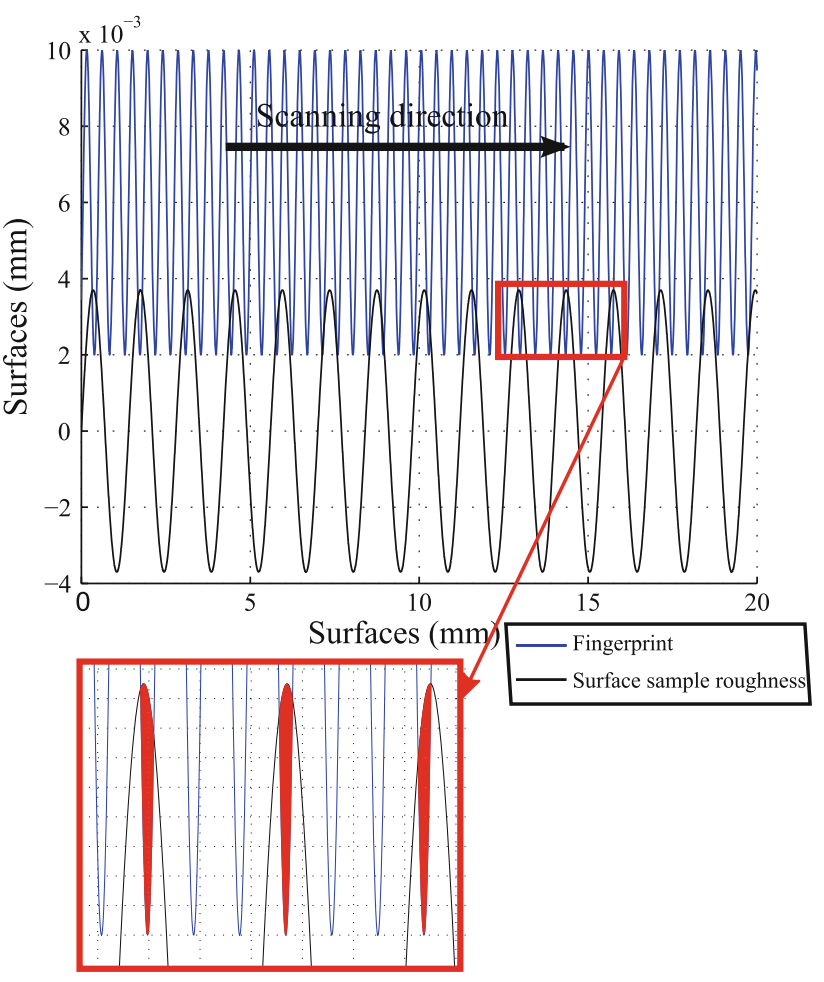

Fig. 10 Sinusoidal surfaces simulated by the model at an instant of time; the red area is the intersection between the two sinusoids at a time $t$, considered to be representative of the skin deformed area "adaptation" to the aluminium profile

At each time step $\left(t_{\mathrm{i}}\right)$, the Matlab script calculates the intersection area $I\left(t_{i}\right)$ between the two sinusoids by calculating the sum of the discrete areas between each pair of points that falls into the intersection zone:

$I\left(t_{i}\right)=\sum_{x \in w 1 \cap w 2}\left(w 1\left(t_{i}, x\right)-w 2(x)\right) \cdot \Delta x$

The time signal $I(t)$, which is the integral of the interpenetration areas between the two surfaces, is considered to be representative of the global vibration signal, obtained experimentally by the accelerometer at the fingernail. As for the experimental acceleration signal [32], the FFT of the interpenetration area $I(t)$ has been performed to calculate the frequency spectrum of the signal and the respective frequency peak, which are reported in Fig. 11 as a function of the surface sample wavelength.

At each intersection between fingerprint and sample roughness, the skin "adapts" its geometry to follow the roughness profile, and the global vibrations measured experimentally at the fingernail are considered proportional to the sum of the local deformations at contact. Obviously, this model is not able to reproduce the amplitude of vibration, which is related to contact and material properties.

Using this model, the sliding between the fingertip and the nine surface samples with periodical roughness 


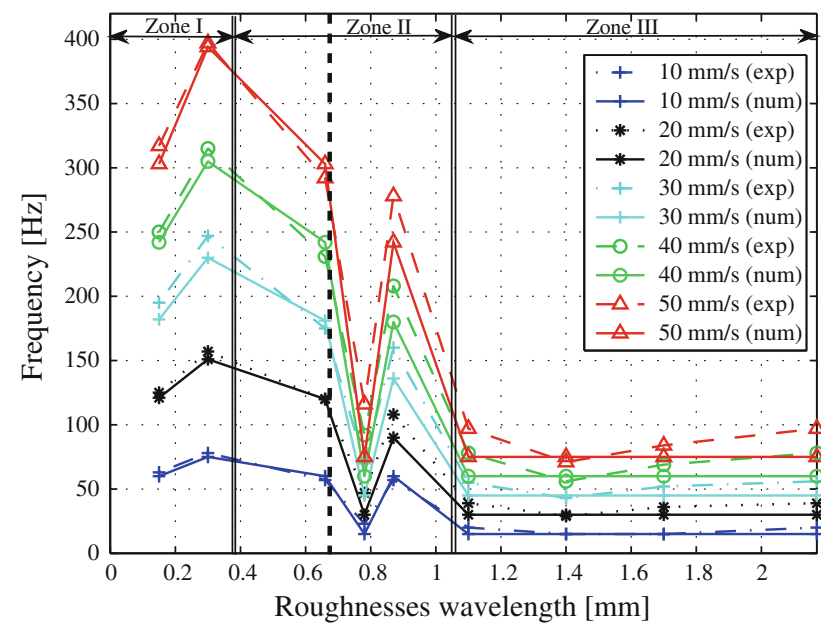

Fig. 11 Comparison between numerical and experimental curves of the relationship between the induced vibration frequency and the roughness width for different scanning speeds. The fingerprint period is equal to $0.66 \mathrm{~mm}$ (black vertical line)

(employed in the experimental measurements) has been simulated for five different scanning speeds (10, 20, 30, 40 and $50 \mathrm{~mm} / \mathrm{s}$ ).

Figure 11 shows the comparison between numerical and experimental results. Each couple of curves (dashed and continuous) plots the frequency ( $y$-axis) of the induced vibrations (experiments) and the signal obtained by the numerical model, as a function of the sample roughness wavelength ( $x$-axis).

The continuous curves represent the frequencies obtained with the numerical model, while the dashed lines correspond to the experimental frequencies at each scanning speed (represented by a different line colour), for a constant contact force of $0.3 \mathrm{~N}$.

Numerical and experimental results are in good agreement. In both the cases, the relationship between the main frequency peaks and the roughness spatial period shows a nonlinear dependence of the frequency peak with respect to the roughness width of the sample, which is due to the presence of the fingertip roughness. From the trend of the induced vibration peaks it is possible to distinguish three different zones in function of the fingerprint wavelength. ZONE I, in which the surface sample wavelength is smaller than the fingerprint one and the frequency peak value depends primarily on the roughness wavelength of the sample. ZONE II, where the surface sample wavelength is comparable to the one of the fingerprint and the frequency peak is function of the ratio between the periods of the sample and of the fingerprint. ZONE III, in which the surface sample wavelength is larger than the fingerprint one and the frequency peak value, depends primarily on the roughness period of the fingerprints. In ZONE III, the measured frequencies are almost constant for all the sample roughness with reference to the same individual. A constant value is also obtained using the numerical model. The slight variation on the experimental values may be ascribed to the shape of the fingerprints that are not perfect sinusoids.

As shown experimentally [40], these results highlight two different possible perception mechanisms of the surface texture as a function of the roughness period. In ZONE I and ZONE II, the spectra of the induced vibrations give information about the surface roughness. In ZONE III, the spectra of the induced vibrations are only function of the fingerprints width. During tests in ZONE III, individuals asserted to perceive the roughness samples as a spatial distribution of the contact pressure between the fingertip and the sample surface, but they could not perceive any difference on the perceived vibrations. On the contrary, when scanning surface samples of ZONE I and ZONE II, subjects could not perceive any information about the pressure spatial distribution, but they did perceive the different roughness of the samples by the sensation of local vibrations at the fingertip/sample interface [32].

The frequency peaks found with the numerical model are in a very good agreement with the experimental ones, even if the real fingerprints and the sample roughness are not perfectly sinusoidal.

The slight difference between experimental and numerical frequencies obtained in ZONE III is due to the differences on the fingerprint profile: real fingerprints are not perfectly sinusoidal and their distribution and orientation is not uniform on the fingertip. Consequently, during contact, the effective fingerprint roughness period differs slightly from the one introduced in the model, which is the mean value obtained from the fingerprints profile.

Thus, the model allows to ascribe the experimental trend of the frequency peaks with respect to the surface texture to the geometrical properties of the surface roughness, highlighting the role of fingerprints in the tactile perception mechanism. In fact, the agreement between the experimental results and those obtained by the simplified model, which accounts only for the geometrical properties of fingerprints and surface sample, allows to assert that the frequency information perceived by the mechanoreceptors is mainly function of the surface roughness profile and it is filtered by the fingerprints geometry.

On the other hand, this simplified numerical model does not provide any information about the vibration amplitude because no material or contact properties are accounted for.

\subsection{Effect of Contact Load}

Another aspect to consider when investigating the induced vibration spectra is the effect of contact load. In order to experimentally analyse its effect on the amplitude of the 
induced vibrations, tests have been developed scanning with the finger on a periodic roughness sample at three different scanning speeds $(10,20$ and $30 \mathrm{~mm} / \mathrm{s})$ for different loads ranging from 0.1 to $1.8 \mathrm{~N}$.

Figure 12 collects the amplitude of the main acceleration peaks in the FFT spectra, obtained at each scanning speed as a function of the contact load. The surface sample used for this analysis is a periodic one, with roughness width equal to $0.3 \mathrm{~mm}$ and mean roughness of $11 \mu \mathrm{m}$. The amplitude values, indicated by markers, are joined by dashed lines and their colour indicates the relative scanning speed (the blue line corresponds to $10 \mathrm{~mm} / \mathrm{s}$, the black one to $20 \mathrm{~mm} / \mathrm{s}$, and the red one to $30 \mathrm{~mm} / \mathrm{s}$ ).

When the contact load is between 0.3 and $1 \mathrm{~N}$, the amplitude of the induced vibrations is almost constant for the same scanning speed, while for larger values of the contact load, the induced vibration amplitude increases as the load does.

The obtained results agree with the neurophysiologic and psychophysical studies $[8,27]$ presented in the literature, asserting that people can discriminate forms and textures of commonly used objects with a high resolution, and that the tactile pattern recognition is independent of contact forces ranging from 0.2 to $1 \mathrm{~N}$ [8]. On the contrary, for higher force values, the normal load applied by the fingertip on the object surface influences the perceived roughness [27]; higher applied forces correspond to higher perceived roughness. This different behaviour was ascribed to both the vibration magnitude increase and the change in contrast in the roughness pattern in contact. The presented results, obtained by the analysis of the induced vibration spectra, highlight the agreement between the perceived roughness and the vibration amplitude.

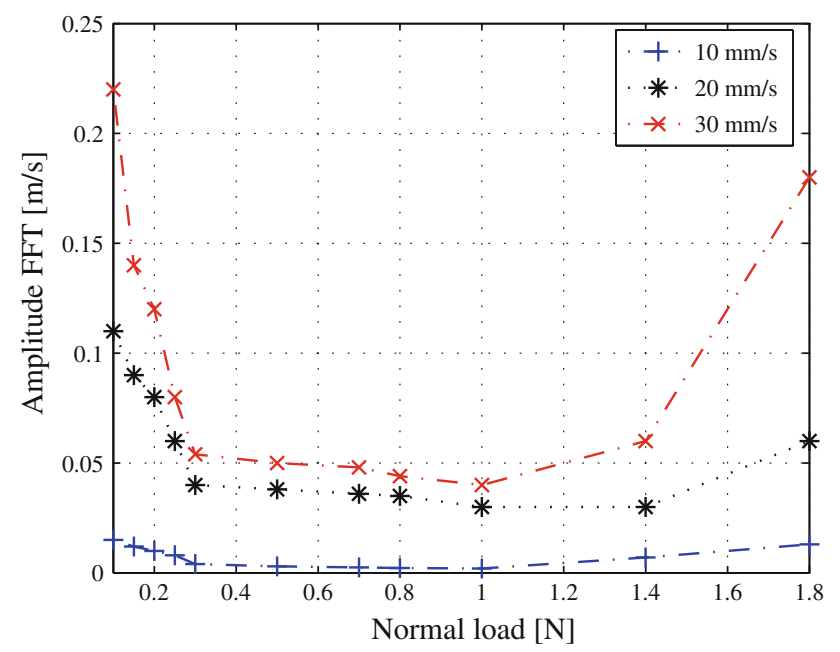

Fig. 12 Relationship between the amplitude of the induced vibrations and the normal load applied during scanning for three different scanning speeds

\subsection{Effect of Fabric Features}

More complex, with respect to the spectra obtained scanning on periodical roughness, are the vibration spectra obtained when scanning textiles [32], due to the complexity of the surface roughness (texture and wire features), the non-isotropic behaviour of the material, and the compliance of the material that cannot be neglected with respect to the skin.

The spectrum obtained scanning a textile with the fingertip (Fig. 13) shows both a well-defined frequency peak, characterising surfaces with periodical roughness, and a larger frequency distribution, which is characteristic of the isotropic roughness of surfaces [32].

These two components are always present in the spectrum obtained scanning on fabric samples, but their relative contribution to the vibration spectra depends on the structure of the textile. Specifically, the frequency peak is related to the periodicity of the fabric texture, while the larger frequency distribution is related to the roughness of the wires composing the texture.

For the tissue shown in Fig. 13, the texture width periodicity is $2.2 \mathrm{~mm}$ (red arrow), which is greater than the fingerprint width, and hence, as expected, the frequency peak of the induced vibrations is the one calculated for the fingerprint width (as the ratio between the scanning speed and the fingerprint width).

The fabric represented in Fig. 14 does not show a welldefined periodic texture, and it is characterised by long

(a)
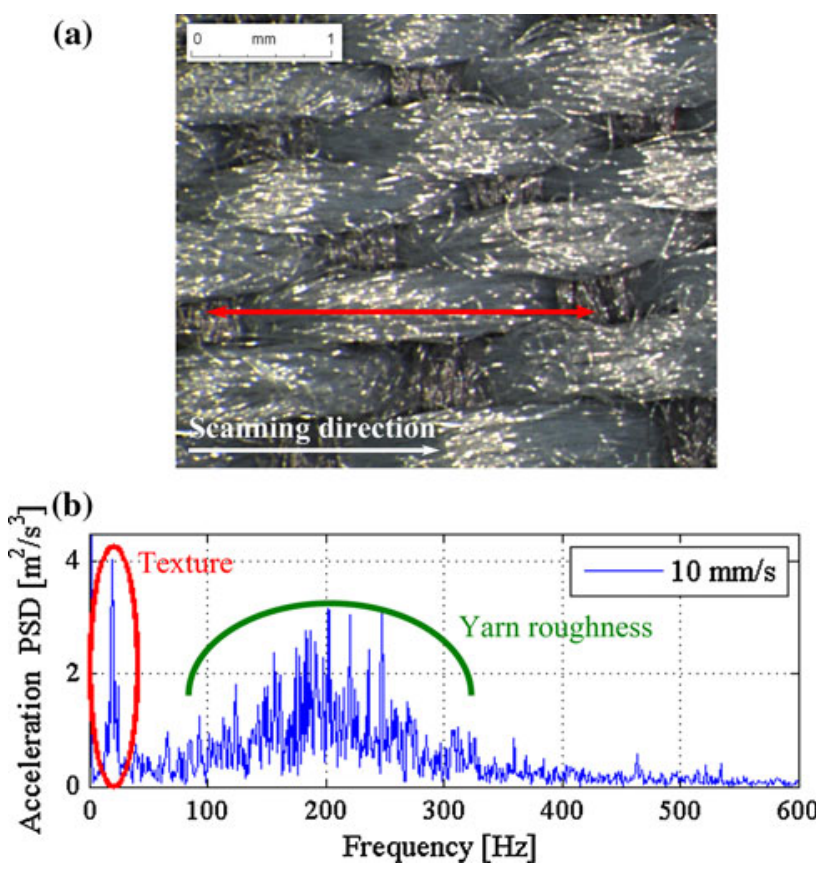

Fig. 13 a Touched tissue. b Acceleration PSD of the signal obtained by the accelerometer 


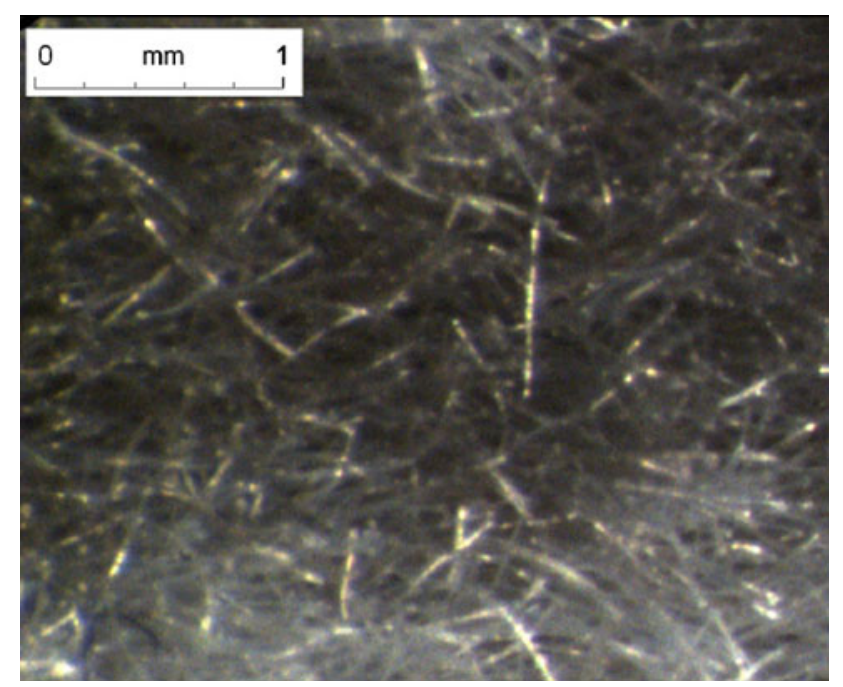

Fig. 14 Example of fabric with a high hairiness and without a welldefined texture

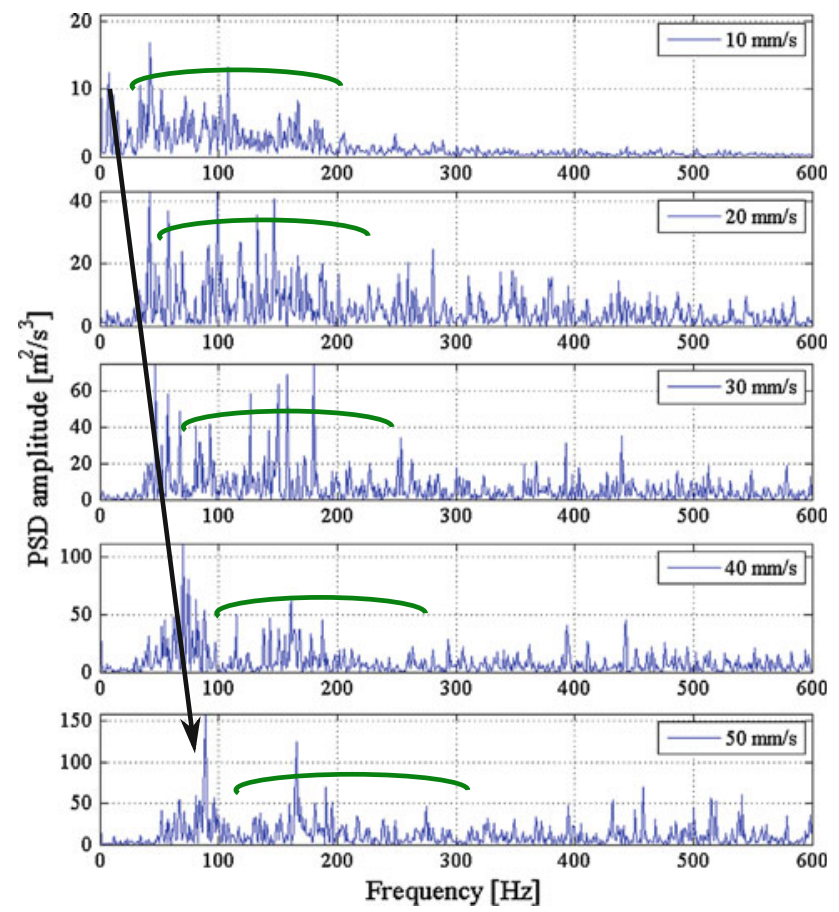

Fig. 15 The acceleration PSD magnitude for the signals obtained from the accelerometer employing the tissue sample in Fig. 14. The black arrow represents the position of the peak corresponding to the fingerprint period, while the green curve covers the frequency distribution given by the yarn roughness (Color figure online)

wires oriented without a preferential direction. The vibration spectrum (Fig. 15), obtained when scanning this fabric with the fingertip, is mainly characterised by a large frequency distribution given by the yarns that provide an isotropic-like roughness of the surface.

In Fig. 15, it is still possible to distinguish the frequency peak due to the fingerprint roughness by means of the large frequency distribution due to the fabric hairiness. Nevertheless for this fabric sample, the contribution of the frequency peak due to the fingerprints is comparable in amplitude to the rest of the spectrum. Its relative contribution, with respect to the large frequency distribution, increases as the scanning speed increases. This behaviour could be ascribed to the mutual compliance of the two surfaces and to the accommodation mechanisms of the textile during the scanning of the fingertip.

Considering the fabric in Fig. 16, characterised by a well-defined texture and with a high yarn compactness, the components given by texture periodicity on the vibration spectra are dominant.

The texture of this fabric is characterised by two fundamental periods (Fig. 16) that are detectable in the corresponding vibration spectra in Fig. 17. Each spectrum presents three main vibration peaks corresponding to the fingerprints period and to the two periods of the fabric texture.

A different behaviour of the amplitude of the peaks can be observed as a function of the scanning speed: while the frequency peak due to the finer texture period (higher frequency) dominates at higher speeds, the amplitude of the vibrations due to a larger texture period (lower frequency) dominates for low scanning speeds. This different behaviour could be ascribed to the accommodation of the textile fibres, occurring during the scanning, that changes the contact pattern. The frequency peak due to the fingerprints, which in this case are stiffer than the scanned surface, stays in the spectrum for all the scanning speeds.

For this fabric, the wires are rewound on themselves conferring a small yarn roughness; as a consequence, the contribution of the large frequency distribution due to the

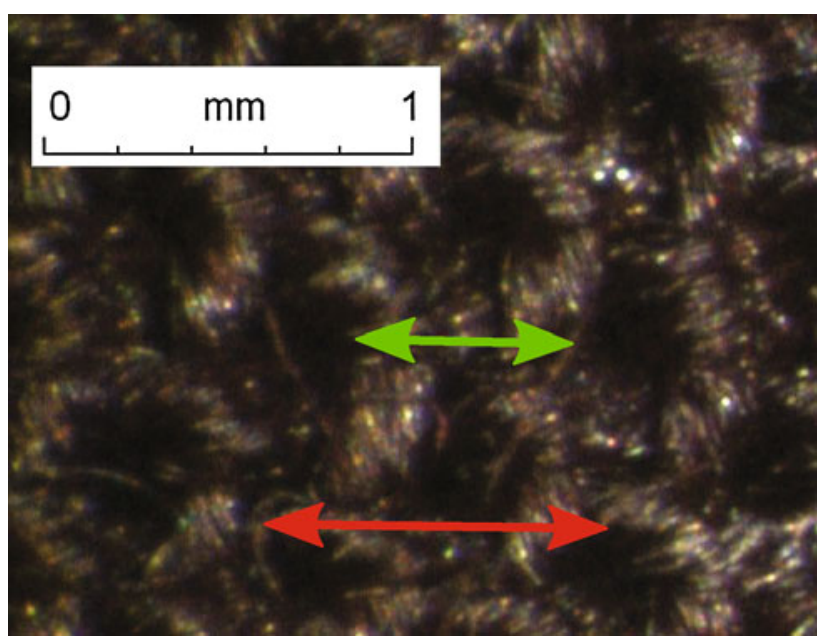

Fig. 16 Example of fabric texture with two texture periods (highlighted by red and green arrows), one of $0.87 \mathrm{~mm}$ and the other of $0.5 \mathrm{~mm}$ in width (Color figure online) 


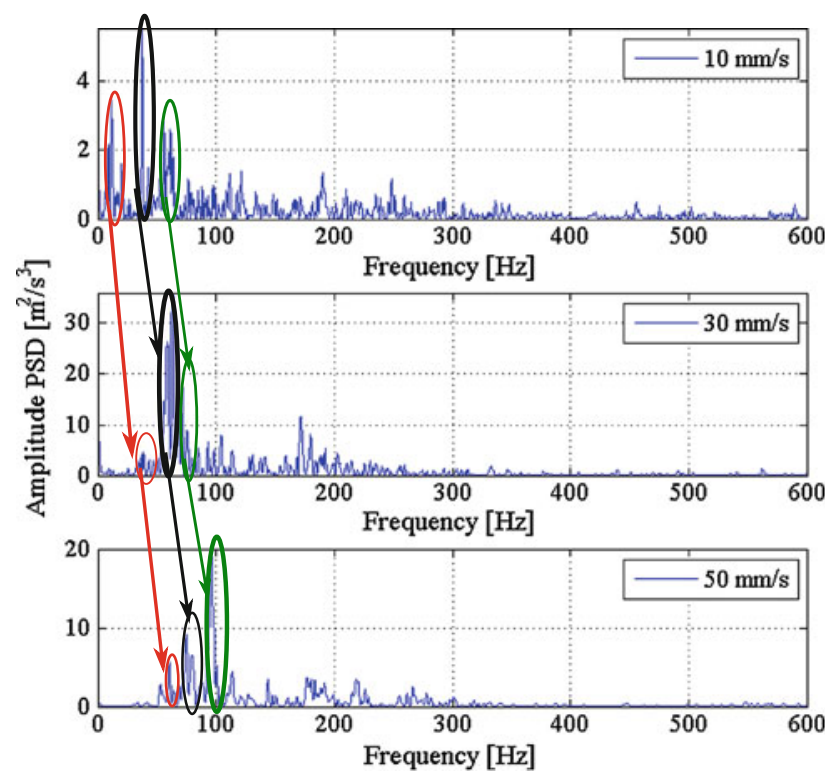

Fig. 17 Acceleration PSD of the signals obtained from the accelerometer employing the fabric sample in Fig. 16. The red arrow represents the position corresponding to the frequency peak due to the larger texture period. The black arrow shows the frequency peak position due the fingerprints, and the green arrow shows the one due to the smaller texture period (Color figure online)

yarn roughness is small compared to the contribution of the frequency peaks given by fingerprints and texture periods.

For every fabric sample, like for surfaces with periodic roughness, it is possible to observe that as the scanning velocity increases the amplitude of the vibration also does and the induced vibrations move to higher frequencies.

\section{Conclusions}

The mechanoreceptors convert the skin stress-state and the vibrations induced by scanning a surface into electric impulses that are then sent to the brain.

To understand the relationship between surface characteristics and tactile sensation, it is necessary to investigate the friction coefficient and the vibrations induced by the fingertip scanning on the object surface by reproducing the contact and measuring vibrations and contact forces.

This article describes the results of an experimental campaign to highlight the effect of scanning speed, contact load, and surface texture on induced vibration and friction coefficient obtained using an innovative test bench, the TriboTouch set-up.

The analysis of the friction coefficient obtained from the sliding contact between fingertip and aluminium samples has proven, in agreement with the literature and the Wolfram theory for the contact between distorting surfaces, a decreasing friction coefficient with respect to the increase of the contact load. A slight decreasing trend with respect to the scanning speed, especially at lower contact force, has been found too.

The decrease of the friction coefficient with the increase of the scanning speed is more significant for the contact between fingertip and fabrics. Moreover, the larger the hairiness of the fabric is, the larger the variations of the friction coefficient with respect to the scanning speed. This behaviour may be related to the mutual compliance of the surfaces in contact and to the accommodation mechanisms of the yarns during the scanning of the fingertip.

A previous work [32] showed the relationship between vibration frequency trend and surface sample roughness wavelength, highlighting the agreement of the experimental results with the "duplex perception model" presented in neurophysiologic literature. Specifically, the experimental results show the possibility to distinguish among three different vibration behaviours on the basis of the relationship between the surface sample roughness width and the one of the fingerprint, suggesting that the transition from a vibrational code (for fine textures) to a spatial one (for coarse textures), affirmed by the "duplex model", is related to the fingerprint width. In this paper a simple numerical model reproducing the interaction between two sinusoidal surfaces in relative motion has been presented. The good agreement with the experimental results and the fact that the model only accounts for the geometrical characteristics of the scanning between fingerprints and surface roughness (no material and contact parameters are accounted for) allow to ascribe the frequency trend of the induced vibrations to the filtering role of the fingerprints pattern.

The analysis of the vibration detected on the finger when scanning on fabric samples has shown spectra characterised by well-defined peaks related to the fabric texture periodicity and a large frequency distribution related to the fabric yarn roughness. These two components are always present in the spectra, but their contribution depends on the fabric features.

The comparison between the spectra obtained on different fabric samples suggests the possibility to distinguish the relative contribution of the yarn roughness and of texture periods. When the yarn roughness is dominant with respect to the texture periods, its contribution to the vibration spectra is larger and comparable in magnitude to the one given by the fingerprints period. On the contrary, its contribution to the vibration spectra is lower for fabric with well-defined texture and low hairiness.

The shown features of the induced vibration spectra, when scanning a textile, open a new interesting domain of investigation to link the two components of the spectra with the quality perception when touching a textile.

Further investigations and statistical campaigns are needed to relate spectra characteristics with perception. In 
particular, the influence of the relative amplitude and of the position (in frequency) of the two spectrum contributions when touching fabrics and of the textile accommodation during fingertip scanning need to be investigated.

\section{References}

1. Johansson, R. S.: Tactile sensibility in man. A quantitative study of the population of mechanoreceptive units in the glabrous skin area of the hand. Umea University Medical Dissertations, New Series No. 35 (1978)

2. Lumpkin, E. A., Caterina, M. J.: Mechanisms of sensory transduction in the skin. Nature 445 (2007). doi:10.1038/nature05662

3. Lynn, B., Perl, E.R.: Afferent mechanisms of pain. In: Kruger, L. (ed.) Pain and Touch, pp. 213-241. Academic Press, San Diego (1996)

4. Spray, D.C.: Cutaneous temperature receptors. Annu. Rev. Physiol. 48, 625-638 (1986)

5. Johnson, K.O.: The roles and functions of cutaneous mechanoreceptors. Curr. Opin. Neurobiol. 11, 455-461 (2001)

6. Bolanowski, S.J., Gescheider, G.A., Verrillo, R.T., Checkosky, C.M.: Four channels mediate the mechanical aspects of touch. J. Acoust. Soc. Am. 84, 1680-1694 (1988)

7. Maeno, T., Kobayashi, K., Yamazaki, N.: Relationship between the structure of human finger tissue and the location of tactile receptors. Bull. JSME Int. J. 41(1C), 4-100 (1998)

8. Johnson, K.O., Yoshioka, T., Vega-Bermudez, F.: Tactile functions of mechanoreceptive afferents innervating the hand. J. Clin. Neurophysiol. 17, 539-558 (2000)

9. Jenmailm, P., Bierznierks, I., Goodwin, A.W., Johansson, R.S.: Influence of object shape on responses of human tactile afferents under conditions characteristic of manipulation. Eur. J. Neurosci. 18, 164-176 (2003)

10. Goodwin, A.W., John, K.T., Marceglia, A.H.: Tactile discrimination of curvature by humans using only cutaneous information from the fingerpads. Exp. Brain Res. 86, 663-672 (1991)

11. Goodwin, A.W., Wheat, H.E.: Magnitude estimation of contact force when objects with different shapes are applied passively to the fingerpad. Somatosens. Mot. Res. 9, 339-344 (1992)

12. Vega-Bermudez, F., Johnson, K.O.: SA I and RA receptive fields, responses variability, and population responses mapped with a probe array. J. Neurophysiol. 81, 2701-2710 (1999)

13. Talbot, W.H., Darian-Smith, I., Kornhuber, H., Mountcastle, V.B.: The sense of flutter-vibration: comparison of the human capacity with response patterns of mechanoreceptive afferents from the monkey hand. J. Neurophysiol. 31, 301-334 (1968)

14. Brisben, A.J., Hsiao, S.S., Johnson, K.O.: Detection of vibration transmitted through an object grasped in a hand. J. Neurophysiol. 81, 1548-1558 (1999)

15. Bolanowski, S.J., Gescheider, G.A., Verrillo, R.T.: Hairy skin: psychophysical channels and their physiological substrates. Somatosens. Mot. Res. 11, 279-290 (1994)

16. Olausson, H., Wessberg, J., Kakuda, N.: Tactile directional sensibility: peripheral neural mechanisms in man. Brain Res. 866, 178-187 (2000)

17. Edin, B.B., Westling, G., Johansson, R.S.: Independent control of human fingertip forces at individual digits during precision lifting. J. Physiol. 487, 243-251 (1995)

18. Collins, D.F., Prochazka, A.: Movement illusions evoked by ensemble cutaneous input from the dorsum of the human hand. J. Neurophysiol. 496, 857-871 (1996)

19. Hollins, M., Goble, A.: Perception of the length of voluntary movements. Somatosens. Res. 5, 335-348 (1988)
20. Hollins, M., Bensmaia, S. J., Risner, R.: The duplex theory of tactile texture perception. In: Proceedings of Fourteenth Annual Meeting of the International Society for Psychophysics, pp. 115-120 (1998)

21. Hollins, M., Bensmaia, S.J., Karlof, K., Young, F.: Individual differences in perceptual space for tactile textures: evidence from multidimensional scaling. Percept. Psychophys. 62, 1534-1544 (2000)

22. Hollins, M., Risner, R.: Evidence for the duplex theory of tactile texture perception. Percept. Psychophys. 62(4), 695-705 (2000)

23. Hollins, M., Bensmaia, S.J., Wahburn, S.: Vibrotactile adaptation impairs discrimination of fine, but not coarse, textures. Somatosens. Mot. Res. 18, 253-262 (2001)

24. Hollins, M., Fox, A., Bishop, C.: Imposed vibration influences perceived tactile smoothness. Perception 29, 1455-1465 (2000)

25. Hollins, M., Lorenz, F., Harper, D.: Somatosensory coding of roughness: the effect of texture adaptation in direct and indirect touch. J. Neurosci. 26, 5582-5588 (2006)

26. Gescheider, G.A., Wright, J.H.: Effects of sensory adaptation on the form of the psychophysical magnitude function for cutaneous vibration. J. Exp. Psychol. 77, 308-313 (1968)

27. Lederman, S.J., Taylor, M.M.: Fingertip force, surface geometry, and perception of roughness by active touch. Percept. Psychophys. 12(5), 401-408 (1972)

28. Lederman, S.J., Loomis, J.M., Williams, D.A.: The role of vibration in the tactual perception of roughness. Percept. Psychophys. 32(2), 109-116 (1982)

29. Lederman, S.J.: Tactual roughness perception: spatial and temporal determinants. Can. J. Psychol. 37(4), 498-511 (1983)

30. Bensmaia, S.J., Hollins, M.: The vibrations of texture. J. Somatosens. Mot. Res. 20(1), 33-43 (2003)

31. Scheibert, J., Leurent, S., Prevost, A., Debregeas, G.: The role of fingerprints in the coding of tactile information probed with a biomimetic sensor. Science 323(5920), 1503-1506 (2009). doi: 10.1126/science. 1166467

32. Fagiani, R., Massi, F., Chatelet, E., Berthier, Y., Akay, A.: Tactile perception by friction induced vibrations. Tribol Int. 44(10), 1100-1110 (2011)

33. Tang, W., Ge, S., Zhu, H., Cao, X., LI, N.: The Influence of normal load and sliding speed on frictional properties of skin. J. Bionic Eng. 5, 33-38 (2008)

34. Asserin, J., Zahouani, H., Humbert, Ph., Couturaud, V., Mougin, D.: Measurements of the friction coefficient of the human skin in vivo. Quantification of the cutaneous smoothness. Colloids Surf. 19, 1-12 (2000)

35. Derler, A., Schrade, U., Gerhardt, L.C.: Tribology of human skin and mechanical skin equivalents in contact with textiles. J. Wear 263, 1112-1116 (2007)

36. Sivamani, R.K., Goodman, J., Gitis, N., Maibach, H.I.: Friction coefficient of skin in real-time. Skin Res. Technol. 9, 235-239 (2003)

37. Lobontiu, N.: Compliant Mechanisms Design of Flexure Hinges. CRC Press, Boca Raton (2002)

38. Fagiani, R., Massi, F., Chatelet, E., Berthier, Y., Sestieri, A.: Experimental analysis of friction induced vibrations at the finger contact surface. In: Proceedings of the Institution of Mechanical Engineers. J. Eng. Tribol. (2009). doi:10.1243/13506501JET722

39. Tang, W., Bhushan, B.: Adhesion, friction and wear characterization of skin and skin cream using atomic force microscope. Colloids Surf. B 76, 1-15 (2010)

40. El-Shimi, A.F.: In vivo skin friction measurements. J. Soc. Cosmet. Chem. 28, 37-51 (1977)

41. Koudine, A., Barquins, M., Barquins, M., Anthoine, P.H., Aubert, L., Leveque, J.L.: Friction properties of skin: proposal of a new approach. Int. J. Cosmet. Sci. 22, 11-20 (2000) 
42. Han, H. Y., Shimada, A., Kawamura, S.: Analysis of friction on human fingers and design of artificial fingers. In: Proceedings of the 1996 IEEE International Conference on Robotics and Automation Minneapolis, Minnesota, April (1996)
43. Koc, I. M., Aksu, C.: Tactile sensing of constructional differences in fabrics with a polymeric fingertip. Tribol Int (2012). http://dx. doi.org/10.1016/j.triboint.2012.04.021 